\title{
A new method for isomorphism identification of planetary gear trains
}

\author{
Wei Sun ${ }^{1,2}$, Ronghe $\mathbf{L i}^{1,2}$, Jianyi Kong ${ }^{1,2}$, and Anming $\mathbf{L i}^{1,2}$ \\ ${ }^{1}$ Key Laboratory of Metallurgical Equipment and Control Technology, \\ Wuhan University of Science and Technology, 430080 Wuhan, China \\ ${ }^{2}$ Hubei Key Laboratory of Mechanical Transmission and Manufacturing Engineering, \\ Wuhan University of Science and Technology, 430080 Wuhan, China
}

Correspondence: Wei Sun (sw@wust.edu.cn)

Received: 25 May 2020 - Revised: 4 October 2020 - Accepted: 14 December 2020 - Published: 18 February 2021

\begin{abstract}
Planetary gear trains (PGTs) are widely used in machinery such as vehicles, pulley blocks, wrist watches, machine tools, and robots. During the process of structural synthesis of PGTs using graph theory, isomorphism identification of graphs is an important and complicated problem. The reliability of the isomorphism detection method directly determines the accuracy of the synthesis result. In this paper, a novel isomorphism identification method for PGTs is proposed. First, a new weighted adjacent matrix is presented to describe the topological graph of PGTs, which has is unique in describing the structure of PGTs. Then, the weighted distance matrix is proposed and the sum of the matrix is obtained, which can determine whether the planetary gear trains is isomorphic or not. Eventually, the examples demonstrate that this new method can be accurately and effectively performed.
\end{abstract}

1

\section{Introduction}

Planetary gear trains (PGTs) include central gears and planetary gears rotating around them. A gear train mechanism has the advantages of high transmission efficiency, large transmission power range, accurate transmission ratio, and strong working reliability. It is the most widely used transmission mechanism at present. In particular, the planetary gear trains also take into account the advantages of small space, low weight, and high rotation efficiency. PGTs are widely used in machine transmissions, robot reducers, vehicle transmissions, gantry cranes, electric tools, and other transmissions. In the past 50 years, the concept of graph theory has been applied to synthesize PGTs (del Castillo, 2002; Hsu and Hsu, 1997; Ravisankar and Mruthyunjaya, 1985; Yan et al., 2006; Shanmukhasundaram et al., 2019b; Tsai, 1987; Tsai and Lin, 1989; Xie et al., 2015a, b; Yang and Ding, 2019), and the synthesis and analysis of planetary gear trains have become a hot topic in mechanism research. At present, the research on mechanism type synthesis mainly focuses on planar kinematic chains (Ding et al., 2010, 2011, 2012, 2013,
2016; Yang et al., 2018). The geometric axis of at least one gear in the planetary gear trains is rotated around the fixed axis of the other gear during transmission. Hsu and Lam (1992) proposed a new graph representation to represent the kinematic structure of a planetary spur gear train efficiently. Yang et al. (2018) proposed a novel displacement graph and a canonical displacement graph model to represent the structure of planetary gear trains. Shanmukhasundaram et al. (2019a, 2021) presented graph-theory-based methods for the detection of degenerate epicyclic gear trains (EGTs) graphs among the enumerated collection. Isomorphism identification of planetary gear trains is an essential step in type synthesis. Graph theory is often used to judge the isomorphism of simple joint and multiple joints kinematic chains, which are also used in planetary gear trains. The accuracy of the isomorphism determination method directly affects the accuracy of the structural synthesis result of planetary gear trains. The establishment of an efficient and accurate isomorphism determination method has always been a hot topic in the field of mechanism. 
In the last few decades, a lot of research has been done on the isomorphism identification of planetary gear trains. Ravisankar and Mruthyunjaya ( $R$ and S, 1985) proposed the characteristic coefficients of the adjacency matrix method for isomorphism identification of planetary gear trains. Kim and Kwak (1990) introduced a mapping functions method to identify nonisomorphic graphs of epicyclic gear trains, which showed that the edge permutations are from the symmetric group of vertex permutations. Hsu (1994) proposed a structural code method for the identification of the displacement isomorphism of planetary gear trains which can represent the topological structure of planetary gear trains. del Castillo (2002) introduced a method for checking for isomorphisms based on the evaluation of a determinant. Rao (2003) proposed isomorphism identification methods for epicyclic gear trains based on hamming string and a genetic algorithm. Liu et al. (2004) proposed an novel kinematic fractionation concept for the determination of epicyclic gear trains. Yang et al. (2007) presented an algorithm for the identification of the isomorphisms of epicyclic gear mechanism, which improved the efficiency and reliability of the isomorphism identification method. Kamesh et al. (2017) proposed a novel and simple algorithm to eliminate isomorphism chains based on the graph theory. It has been tested on examples from planar kinematic chains with eight links and 1 DOF (degree of freedom), 10 links and 1 DOF, 12 links and 1 DOF, and 15 links and 4 DOF. Yang and Ding (2018a, b, 2019) proposed a fully automatic algorithm to detect and eliminate degenerate planetary gear trains. And the previous perimeter-loopbased isomorphism detection method has been improved to detect isomorphic planetary gear trains. It is applicable for linkage kinematic chains and has been proved to be reliable and efficient. Rai and Punjabi (2019) presented a simple algorithm of links labeling, which was used to find out a binary sequence that provides a maximum binary code. The maxi codes are generated, including binary sequence and binary code, to compare the isomorphisms of planetary gear trains. Xu et al. (2020) proposed a novel isomorphism detection method for the planetary gear transmission structure based on a matrix operation. The various components of the transmission structure and isomorphic structures of the numerous structures are classified.

In this paper, a distance matrix method for isomorphism identification of planetary gear trains is developed. First, a weighted adjacent matrix is proposed to represent the topological structure of planetary gear trains, which can uniquely represent the gear trains, including all information about them. Then, the weighted distance matrix is proposed by an iterative algorithm, and it can determine whether the planetary gear trains are isomorphic or not by sum of this matrix. This isomorphism identification method is simple and reliable and does not require complex computation. Finally, this new isomorphism identification method for PGTs proved to be accurately and effectively performed through a large number of examples.

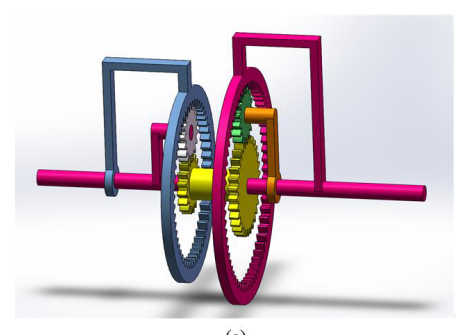

(a)

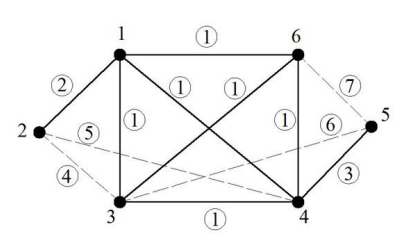

(c)

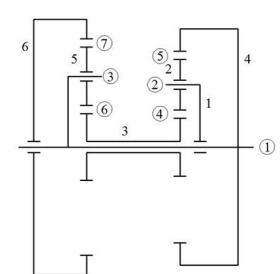

(b)

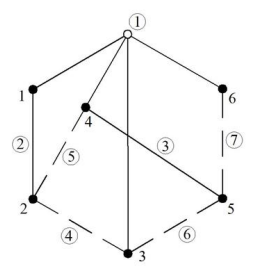

(d)
Figure 1. (a) A 3D model of the Simpson gear train, (b) a schematic diagram, (c) a graphic representation, and (d) bicolor topological graph $a_{1}$.

\section{Representation of the weighted adjacent matrix}

A 3D model of the famous Simpson gear drive system is shown in Fig. 1a. The gear drive system is a 6 bar 1 DOF planetary gear train. Figure $1 b$ is the schematic diagram of a Simpson gear system. Its links and kinematic pairs (revolute pairs and higher pairs) are numbered, respectively. In this paper, the traditional representation of imaginary and real line topology (Hsu and Lam, 1992) is improved. Solid nodes are used to represent links. The real edges represent all the revolute pairs. The gear pairs are indicated by dotted lines. The corresponding topological graph can be obtained from the schematic diagram using the traditional dotted and solid line representation, as shown in Fig. 1c. Considering the planetary gear trains with multiple bars and multiple kinematic pairs, there are many imaginary and real lines represented. In order to simplify the graph, the lines with the same serial number of kinematic pairs in the topological graph are represented by a hollow node, as shown in Fig. 1d. Therefore, all solid nodes connected with the hollow node mean that their corresponding members have the same revolute pair.

A gear train kinematic chain is a collection of links connected by joints, and this link and joint assemblage can be represented by the weighted adjacent matrix. The serial number of the links and kinematic pairs is not limited, and it only needs to be labeled in sequence. The size of the matrix is $n \times n$, and $n$ represents the number of links. The weighted adjacent matrix is expressed as follows:

$$
a_{i, j}=\left\{\begin{array}{l}
0, \text { if } i=j \\
\infty, \text { if link } i \text { is not connected to link } j, \\
\left(100 d_{\max }+d_{\min }\right) / 1000, \text { if link } i \text { is connected } \\
\text { to link } j \text { by geared pair } \\
1+\left(100 d_{\max }+d_{\min }\right) / 1000, \text { if link } i \text { is connected } \\
\text { to link } j \text { by revolute pair }
\end{array},\right.
$$


where, $d_{\max }=\max \left\{d_{i}, d_{j}\right\}$, and $d_{\min }=\min \left\{d_{i}, d_{j}\right\}$. The symbol $d_{i}$ is the degree of link $i$.

$d_{i}=\left\{\begin{array}{l}d_{k}^{\prime}+\left(10 n_{\mathrm{r}}+n_{\mathrm{g}}\right) / 10-1, \text { if link } i \text { is connected } \\ \text { to multiple joint } k \\ \left(10 n_{\mathrm{r}}+n_{\mathrm{g}}\right) / 10, \text { if link } i \text { is not connected } \\ \text { to multiple joint } k\end{array}\right.$,

where, $n_{\mathrm{r}}$ represents the number of revolute pairs in this link. $n_{\mathrm{g}}$ is the number of gear pairs in this link. For instance, the link 6 is connected multiple joint 1 , and the degree of multiple joint 1 is 4 , i.e., $d_{1}^{\prime}=4$. The numbers of revolute pairs and gear pairs are 0 and 1, respectively, i.e., $n_{\mathrm{r}}=0$ and $n_{\mathrm{g}}=1$. Thus, $d_{6}=3.1$.

The weighted adjacent matrix $\mathbf{A}_{a_{1}}$ of the Simpson gear train $a_{1}$ is as follows:

$\mathbf{A}_{a_{1}}=\left[\begin{array}{cccccc}0 & 1.4012 & 1.4032 & 1.4140 & \infty & 1.4031 \\ 1.4012 & 0 & 0.3212 & 0.4112 & \infty & \infty \\ 1.4032 & 0.3212 & 0 & 1.4132 & 0.3212 & 1.3231 \\ 1.4140 & 0.4112 & 1.4132 & 0 & 1.4112 & 1.4131 \\ \infty & \infty & 0.3212 & 1.4112 & 0 & 0.3112 \\ 1.4031 & \infty & 1.3231 & 1.4131 & 0.3112 & 0\end{array}\right]$

\section{The shortest distance matrix}

The shortest distance matrix corresponding to the topological diagram is denoted by the symbol $\mathbf{E}$. The length of the shortest path among all the paths from joint to joint is represented by $\mathbf{E}(i, j)$. The iterative algorithm is utilized to obtain the shortest distance matrix. And the matrices $\mathbf{E}^{(0)}, \mathbf{E}^{(1)}, \mathbf{E}^{(2)} \ldots \mathbf{E}^{(n)}$, and $\left(\mathbf{E}^{(0)}=\left(e_{i j}^{(0)}\right)_{n \times n}=\mathbf{A}\right)$ are constructed.

$\mathbf{E}^{(n)}=\left(e_{i j}^{(n)}\right)_{n \times n}, \quad e_{i j}^{(n)}=\min \left\{e_{i j}^{(n-1)}, e_{i n}^{(n-1)}+e_{n j}^{(n-1)}\right\}$,

where, $e_{i j}^{(n)}$ represents the length of the shortest path from joint $i$ to joint $j$. So, the final matrix $\mathbf{E}^{(n)}$ is the shortest distance matrix. The updating algorithm of the shortest distance matrix is as follows:

Step 1. Initialization, in which the improved adjacency matrix $\mathbf{A}$ is assigned to the initial improved shortest distance matrix E.

Step 2. The shortest distance matrix is updated as $\mathbf{E}(i, j)$. If $\mathbf{E}(i, k)+\mathbf{E}(k, j)<\mathbf{E}(i, j)$, then $\mathbf{E}(i, j)=\mathbf{E}(i, k)+\mathbf{E}(k, j)$

Step 3. If $k=n$, stop. Otherwise, if $k=k+1$, go to Step 2 .

Through the corresponding operation, the shortest distance matrix $\mathbf{E}_{a_{1}}$ of the gear train $a_{1}$ can be achieved as follows:

$$
\begin{aligned}
\mathbf{E}_{a_{1}} & =\left[\begin{array}{cccccc}
0 & 1.4012 & 1.4032 & 1.4140 & 1.7143 & 1.4031 \\
1.4012 & 0 & 0.3212 & 0.4112 & 0.6424 & 0.9536 \\
1.4032 & 0.3212 & 0 & 0.7324 & 0.3212 & 0.6324 \\
1.4140 & 0.4112 & 0.7324 & 0 & 1.0536 & 1.3648 \\
1.7143 & 0.6424 & 0.3212 & 1.0536 & 0 & 0.3112 \\
1.4031 & 0.9536 & 0.6324 & 1.3648 & 0.3112 & 0
\end{array}\right], \\
& {\left[\begin{array}{c}
7.3358 \\
3.7296 \\
3.4104 \\
4.9760 \\
4.0427 \\
4.6651
\end{array}\right] . }
\end{aligned}
$$

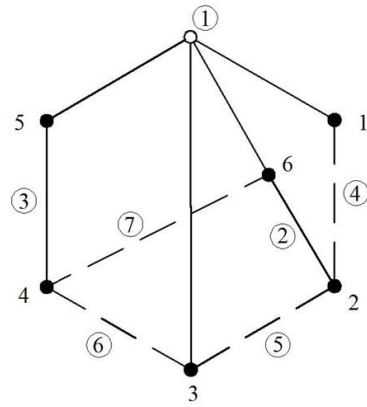

(a)

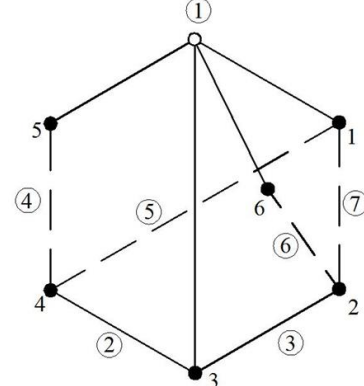

(b)
Figure 2. (a) Bicolor topological graph $a_{2}$ and (b) bicolor topological graph $a_{3}$.

The array of columns on the right-hand side of Eq. (5), which is the sum of the shortest distances from any joint to all other joints in the gear train, is denoted as the sum array. The sum of the shortest distance matrix is $\mathbf{S}_{a_{1}}=28.1596$.

\section{Isomorphism identification}

The process of isomorphism identification is as follows: first, the weighted adjacent matrixes of the planetary gear trains are obtained. If the size of the matrixes is not same, then the planetary gear trains are not isomorphic. Then, the sum array of the planetary gear trains are obtained by shortest distance matrix. If the information is different, the planetary gear trains are not isomorphic. If the information is same, then the planetary gear trains are isomorphic. A total of two 6 bar planetary gear trains are shown in Fig. 2. According to the description of the weighted adjacent matrix in Sect. 2, the expression of the 6 bar planetary gear trains $a_{2}$ and $a_{3}$ is carried out.

The weighted adjacent matrix of the 6 bar planetary gear trains $a_{2}$ and $a_{3}$ are as follows:

$$
\mathbf{A}_{a_{2}}=\left[\begin{array}{cccccc}
0 & 1.4012 & 1.4032 & 1.4140 & \infty & 1.4031 \\
1.4012 & 0 & 0.3212 & 0.4112 & \infty & \infty \\
1.4032 & 0.3212 & 0 & 1.4132 & 0.3212 & 1.3231 \\
1.4140 & 0.4112 & 1.4132 & 0 & 1.4112 & 1.4131 \\
\infty & \infty & 0.3212 & 1.4112 & 0 & 0.3112 \\
1.4031 & \infty & 1.3231 & 1.4131 & 0.3112 & 0
\end{array}\right],
$$

$$
\mathbf{A}_{a_{3}}=\left[\begin{array}{cccccc}
0 & 0.3212 & 1.5032 & 0.3212 & 1.3231 & 1.3231 \\
0.3212 & 0 & 1.5012 & \infty & \infty & 0.3112 \\
1.5032 & 1.5012 & 0 & 1.5012 & 1.5031 & 1.5031 \\
0.3212 & \infty & 1.5012 & 0 & 0.3112 & \infty \\
1.3231 & \infty & 1.5031 & 0.3112 & 0 & 1.3131 \\
1.3231 & 0.3112 & 1.5031 & \infty & 1.3131 & 0
\end{array}\right] .
$$


The shortest distance matrix of the 6 bar planetary gear trains $a_{2}$ and $a_{3}$ are as follows:

$$
\begin{aligned}
& \mathbf{E}_{a_{2}}=\left[\begin{array}{cccccc}
0 & 1.4012 & 1.4032 & 1.4140 & 1.7143 & 1.4031 \\
1.4012 & 0 & 0.3212 & 0.4112 & 0.6424 & 0.9536 \\
1.4032 & 0.3212 & 0 & 0.7324 & 0.3212 & 0.6324 \\
1.4140 & 0.4112 & 0.7324 & 0 & 1.0536 & 1.3648 \\
1.7143 & 0.6424 & 0.3212 & 1.0536 & 0 & 0.3112 \\
1.4031 & 0.9536 & 0.6324 & 1.3648 & 0.3112 & 0
\end{array}\right], \\
& {\left[\begin{array}{l}
7.3358 \\
3.7296 \\
3.4104 \\
4.9760 \\
4.0427 \\
4.6651
\end{array}\right]} \\
& \mathbf{E}_{a_{3}}=\left[\begin{array}{cccccc}
0 & 0.3212 & 1.5032 & 0.3212 & 0.6324 & 0.6324 \\
0.3212 & 0 & 1.5012 & 0.6424 & 0.9536 & 0.3112 \\
1.5032 & 1.5012 & 0 & 1.5012 & 1.5031 & 1.5031 \\
0.3212 & 0.6424 & 1.5012 & 0 & 0.3112 & 0.9536 \\
0.6324 & 0.9536 & 1.5031 & 0.3112 & 0 & 1.2648 \\
0.6324 & 0.3112 & 1.5031 & 0.9536 & 1.2648 & 0
\end{array}\right], \\
& {\left[\begin{array}{l}
3.4104 \\
3.7296 \\
7.5118 \\
3.7296 \\
4.6651 \\
4.6651
\end{array}\right]}
\end{aligned}
$$

From Eqs. (5), (8), and (9), the sums of shortest distance matrixes are $\mathbf{S}_{a_{1}}^{T}=\mathbf{S}_{a_{2}}^{T}=28.1596$ and $\mathbf{S}_{a_{3}}^{T}=27.7116$. So, we can confirm the PGT $a_{1}$ and the PGT $a_{2}$ are isomorphic. The sum array of PGT $a_{3}$ is different. Therefore, PGT $a_{3}$ is not isomorphic.

The greatest advantage of this method is that it is more efficient than other methods without much additional computation. The time complexity of shortest distance matrix method is $\mathbf{O}\left(n^{2}\right)$. The method implements a number to represent a numerical value of a planetary gear train.

\section{Case analysis}

Case 1. Figure 3 shows the topological graph of two 8 bar planetary gear trains. They all contain six gear pairs.

The weighted adjacent matrix of the 8 bar planetary gear trains $b_{1}$ and $b_{2}$ are as follows:

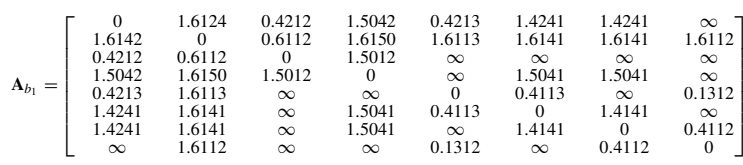

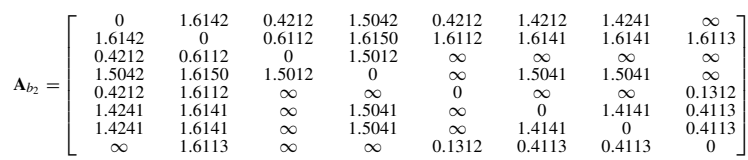

The shortest distance matrix of the 8 bar planetary gear trains $b_{1}$ and $b_{2}$ are as follows:

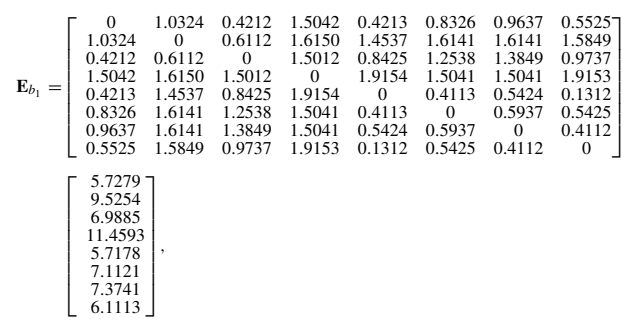

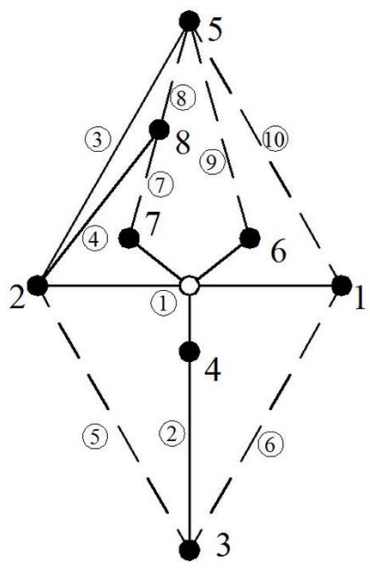

(a)

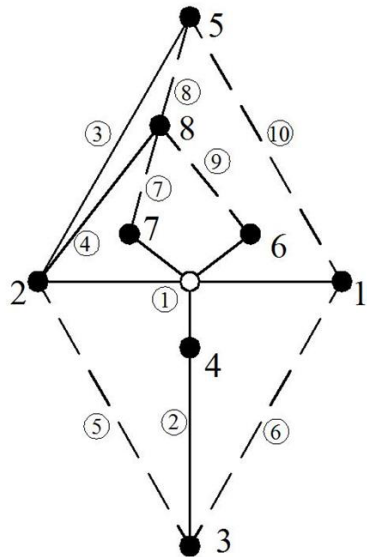

(b)
Figure 3. (a) Bicolor topological graph $b_{1}$ and (b) bicolor topological graph $b_{2}$.

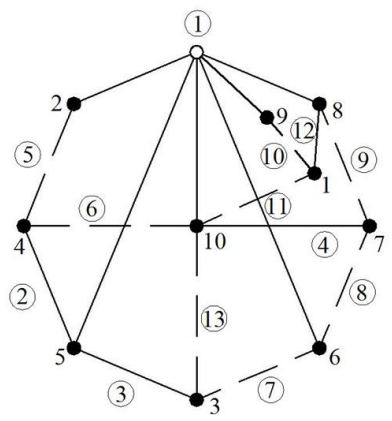

(a)

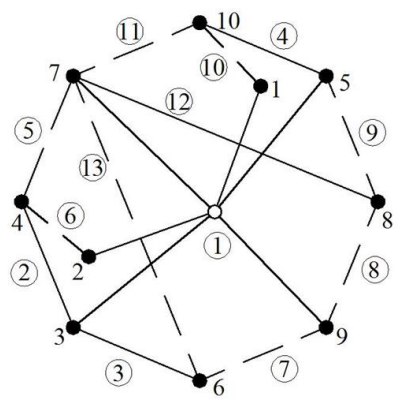

(b)
Figure 4. (a) Bicolor topological graph $c_{1}$ and (b) bicolor topological graph $c_{2}$.

$\mathbf{E}_{b_{2}}=\left[\begin{array}{cccccccc}0 & 1.0324 & 0.4212 & 1.5042 & 0.4212 & 0.9637 & 0.9637 & 0.5524 \\ 1.0324 & 0 & 0.6112 & 1.6150 & 1.4536 & 1.6141 & 1.6141 & 1.5848 \\ 0.4212 & 0.6112 & 0 & 1.5012 & 0.8424 & 1.3849 & 1.3849 & 0.9736 \\ 1.5042 & 1.6150 & 1.5012 & 0 & 1.9254 & 1.5041 & 1.5041 & 1.9154 \\ 0.4212 & 1.4536 & 0.8424 & 1.9254 & 0 & 0.5425 & 0.5425 & 0.1312 \\ 0.9637 & 1.6141 & 1.3849 & 1.5041 & 0.5425 & 0 & 0.8226 & 0.4113 \\ 0.9637 & 1.6141 & 1.3849 & 1.5041 & 0.5425 & 0.8226 & 0 & 0.4113 \\ 0.5524 & 1.5848 & 0.9736 & 1.9154 & 0.1312 & 0.4113 & 0.4113 & 0\end{array}\right]$,

$$
\left[\begin{array}{c}
5.8588 \\
9.5252 \\
7.1194 \\
11.4694 \\
5.8588 \\
7.2432 \\
7.2432 \\
5.98
\end{array}\right] .
$$

From Eqs. (12) and (13), the sums of the shortest distance matrixes are $\mathbf{S}_{b_{1}}^{T}=60.0164$ and $\mathbf{S}_{b_{2}}^{T}=60.298$. Therefore, the two 8 bar planetary gear trains are non-isomorphic.

Case 2. There are a total of two 10 bar planetary gear trains as shown in Fig. 4. They all contain eight gear pairs.

The weighted adjacent matrix of the 10 bar planetary gear trains $c_{1}$ and $c_{2}$ are as follows:

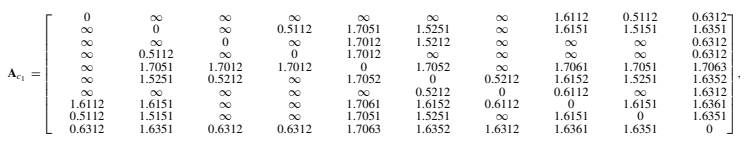


Table 1. Isomorphism determination method of PGTs.

\begin{tabular}{llr}
\hline Method name & Intuitiveness & Example \\
\hline Rao (Rao, 2003) & Half-adjacency string & $160-[22-3(6), 5,3(3)]-3[20-6,3(3), 2(2), 1]$ \\
& & $-3[18-4(3), 3(2)]-[14-5,3(2), 3(1)]$ \\
\hline Yang (Yang et al., 2018) & Characteristic code & $4101011-020100-02001$ \\
& & $-0200-020-00-0$ \\
\hline Kamesh (Kamesh et al., 2017) & Net distance and string & 20 and 20-2(6)-2(4) \\
\hline Rai (Rai and Punjabi, 2019) & Maxi code & $897+32 \mathrm{ee}+18 \mathrm{eI}$ \\
\hline This method & A number & 28.1596 \\
\hline
\end{tabular}

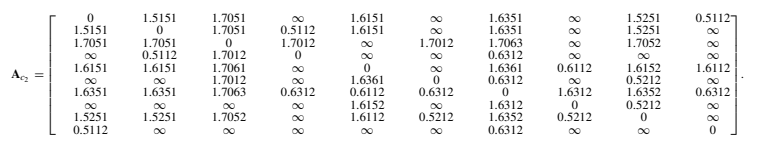

The shortest distance matrix of the 10 bar planetary gear trains $c_{1}$ and $c_{2}$ are as follows:
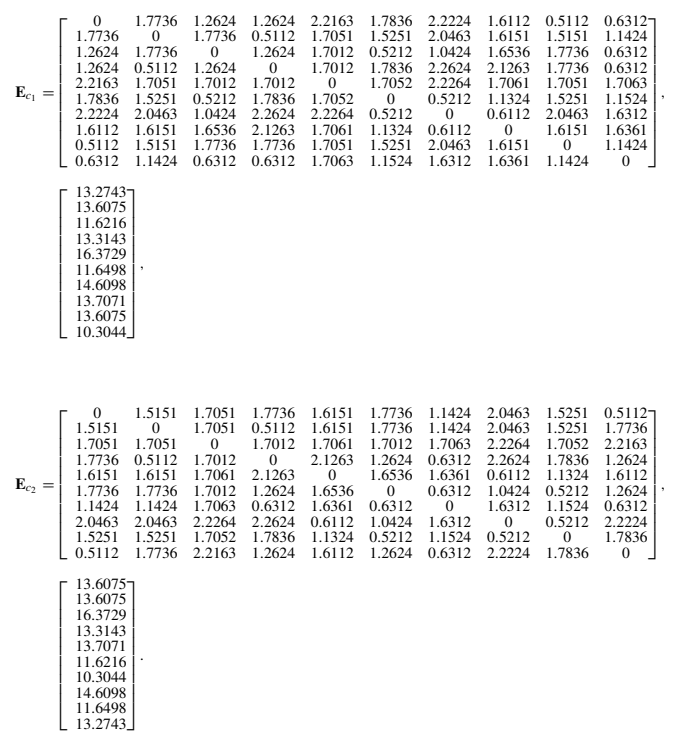

From Eqs. (16) and (17), the sums of shortest distance matrixes are $\mathbf{S}_{c_{1}}^{T}=\mathbf{S}_{\mathrm{c}_{2}}^{T}=132.0692$. So, we can confirm the PGT $c_{1}$ and the PGT $c_{2}$ are isomorphic.

\section{Conclusions}

In this paper, the weighted adjacent matrix is introduced to describe the planetary gear train, which can uniquely represent the structure of the gear train. Then, a novel isomorphism identification method was proposed. This method is both reliable and simple. The time complexity of the shortest distance matrix method is $\mathbf{O}\left(n^{2}\right)$. The greatest advantage of this method is that it is more efficient than other methods without much additional computation. The experimental results provided show the high performance. 
Appendix A

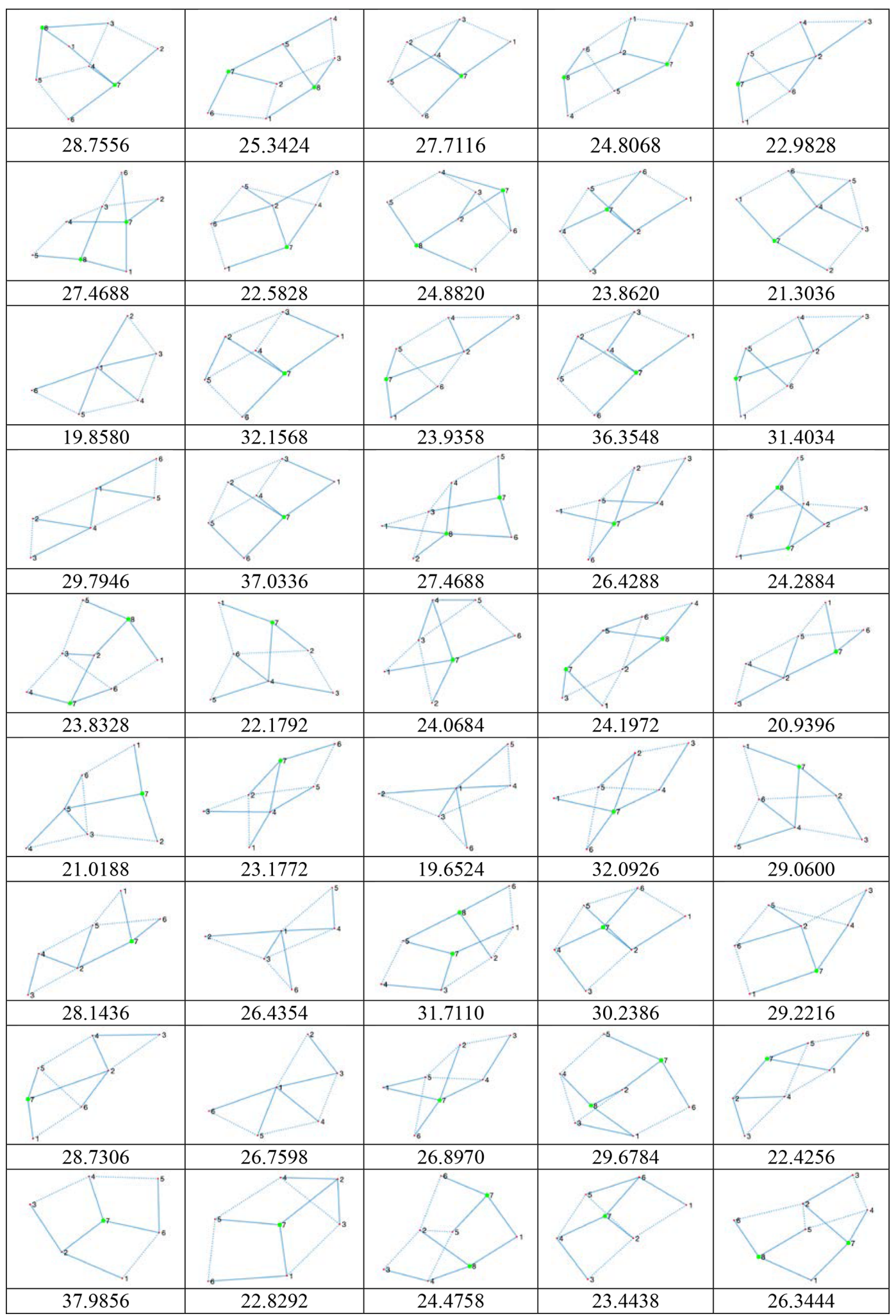

Figure A1. 


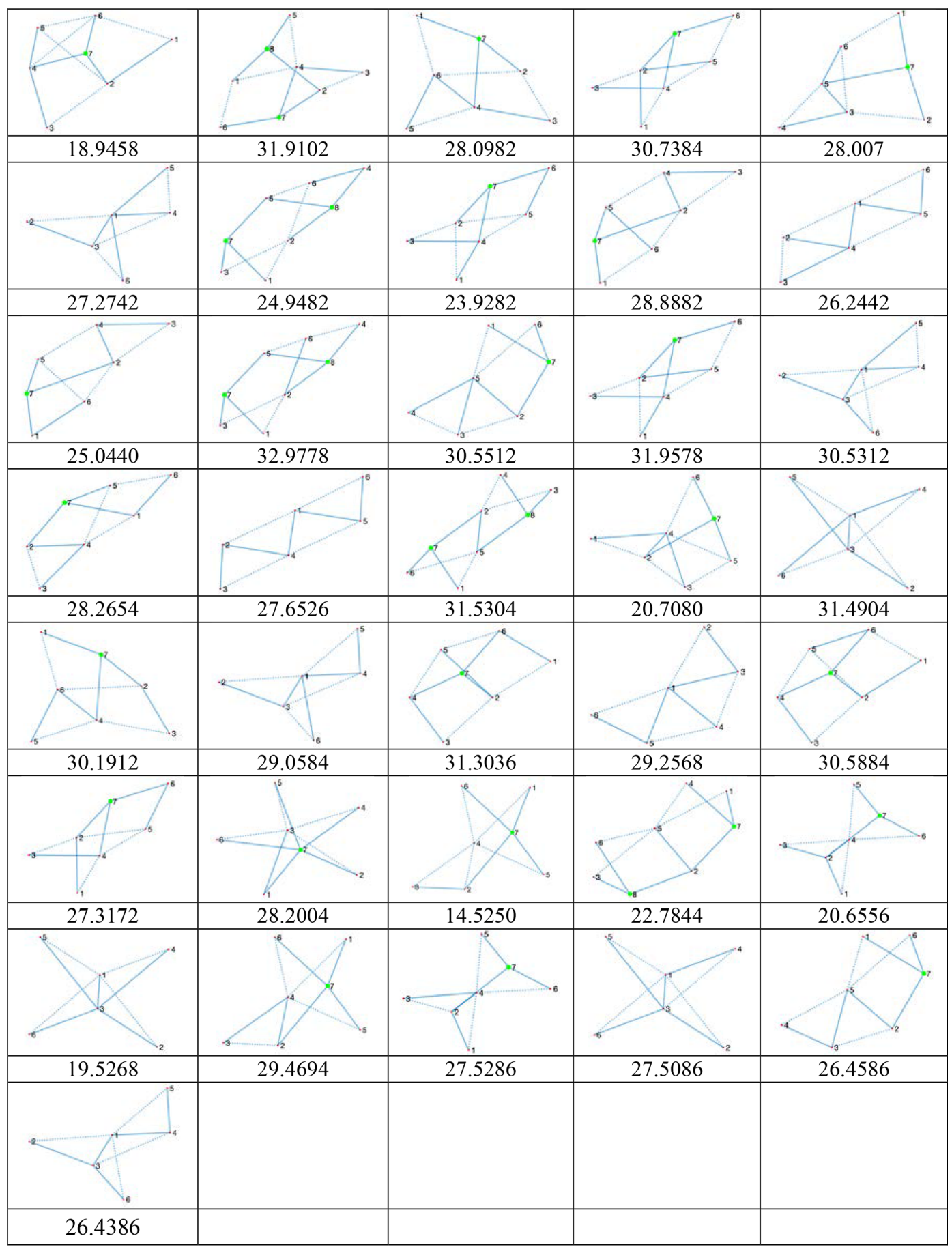

Figure A1. A total of 81 displacement graphs of 6 bar 1 DOF planetary gear trains. 


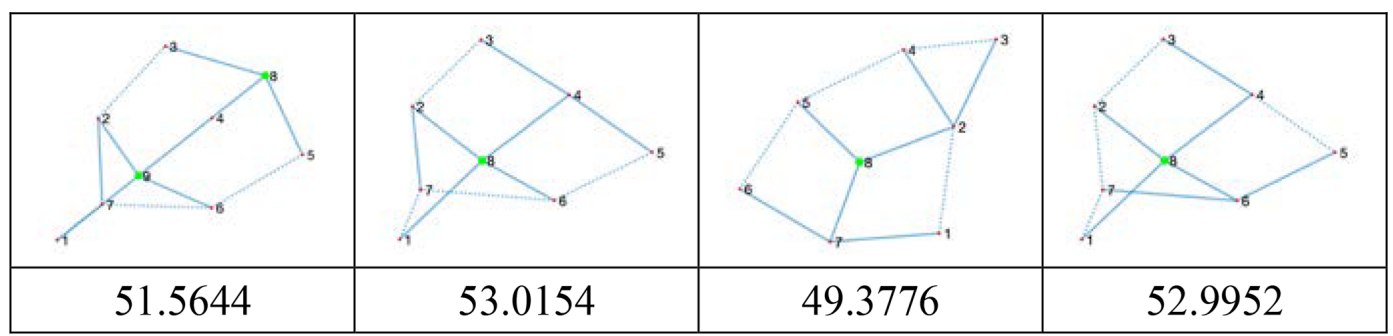

Figure A2. A total of four displacement graphs of 7 bar 2 DOF planetary gear trains.

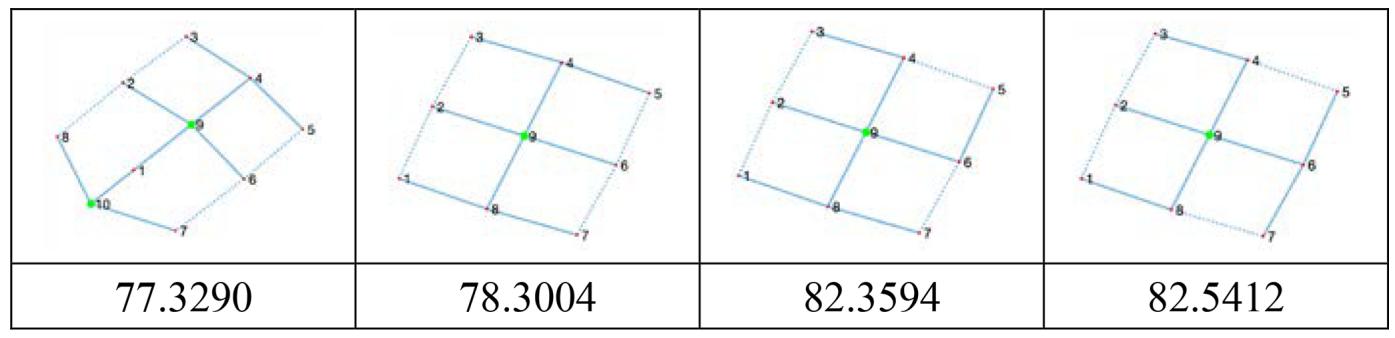

Figure A3. A total of four displacement graphs of 8 bar 3 DOF planetary gear trains. 
Code availability. The code used in this research is available online at https://cloud.189.cn/t/Uryii2MVnmEz (Sun et al., 2021a).

Data availability. The data are available online at https://cloud. 189.cn/t/EJrEN36rQnUv (Sun et al., 2021b).

Author contributions. WS came up with the idea and wrote the paper. RL completed the design and calculations of the experiment. JK took part in a discussion of ideas for the paper. AL was mainly responsible for the construction of isomorphism determination method.

Competing interests. The authors declare that they have no conflict of interest.

Financial support. This research has been supported by the National Natural Science Foundation of China (grant no. 51875418), the Foundation of Hubei Provincial Education Department (grant no. B2020011), and the WUST National Defense Pre-research Foundation (grant no. GF202008).

Review statement. This paper was edited by Guowu Wei and reviewed by two anonymous referees.

\section{References}

Rao, A. C.: A genetic algorithm for epicyclic gear trains, Mech. Mach. Theory, 38, 135-147, 2003.

del Castillo, J. M.: Enumeration of 1-DOF Planetary Gear Train Graphs Based on Functional Constraints, J. Mech. Design, 124, 723-732, 2002.

Ding, H., Zhao, J., and Huang, Z.: Unified structural synthesis of planar simple and multiple joint kinematic chains, Mech. Mach. Theory, 45, 555-568, 2010.

Ding, H., Hou, F., Kecskeméthy, A., and Huang, Z.: Synthesis of a complete set of contracted graphs for planar non-fractionated simple-jointed kinematic chains with all possible DOFs, Mech. Mach. Theory, 46, 1588-1600, 2011.

Ding, H., Cao, W., Kecskeméthy, A., and Huang, Z.: Complete Atlas Database of 2-DOF Kinematic Chains and Creative Design of Mechanisms, J. Mech. Design, 134, 1-10, 2012.

Ding, H., Yang, W., Huang, P., and Kecskeméthy, A.: Automatic structural synthesis of planar multiple joint kinematic chains, J. Mech. Design, 135, 1-12, 2013.

Ding, H., Huang, P., Yang, W., and Kecskeméthy, A.: Automatic generation of the complete set of planar kinematic chains with up to six independent loops and up to 19 links, Mech. Mach. Theory, 96, 75-93, 2016.

Hsu, C.-H. and Lam, K.-T.: A New Graph Representation for the Automatic Kinematic Analysis of Planetary Spur-Gear Trains, J. Mech. Design, 114, 196-200, 1992.

Hsu, C.-H.: Displacement Isomorphism of Planetary, Mech. Mach. Theory, 29, 513-523, 1994.
Hsu, C. H. and Hsu, J. J.: An efficient methodology for the structural synthesis of geared kinematic chains, Mech. Mach. Theory, 32, 957-973, 1997.

Hsu, C. H. and Lam, K. T.: A New Graph Representation for the Automatic Kinematic Analysis of Planetary Spur-Gear Trains, J. Mech. Design, 114, 196-200, 1992.

Kamesh, V. V., Rao, K. M., and Rao, A. B. S.: An Innovative Approach to Detect Isomorphism in Planar and Geared Kinematic Chains Using Graph Theory, J. Mech. Design, 139, 1-45, 2017.

Kim, J. U. and Kwak, B. M.: Application of edge permutation group to structural synthesis of epicyclic gear trains, Mech. Mach. Theory, 25, 563-574, 1990.

Liu, C. P., Chen, D. Z., and Chang, Y. T.: Kinematic analysis of geared mechanisms using the concept of kinematic fractionation, Mech. Mach. Theory, 39, 1207-1221, 2004.

Ravisankar, R. and Mruthyunjaya, T. S.: Computerized synthesis of the structure of geared kinematic chains, Mech. Mach. Theory, 20, 367-387, 1985.

Rai, R. K. and Punjabi, S.: A new algorithm of links labelling for the isomorphism detection of various kinematic chains using binary code, Mech. Mach. Theory, 131, 1-32, 2019.

Rao, A. C.: A genetic algorithm for epicyclic gear trains, Mech. Mach. Theory, 38, 135-147, 2003.

Yan, H.-S., Wang, H.-T., and Liu, J.-Y.: Structural synthesis of novel integrated DC gear motors, Mech. Mach. Theory, 41, 12891305, 2006.

Shanmukhasundaram, V. R., Rao, Y. V. D., and Regalla, S. P.: Algorithms for detection of degenerate structure in epicyclic gear trains using graph theory, J. Braz. Soc. Mech. Sci., 41, 496, https://doi.org/10.1007/s40430-019-2003-5, 2019a.

Shanmukhasundaram, V. R., Rao, Y. V. D., and Regalla, S. P.: Enumeration of displacement graphs of epicyclic gear train from a given rotation graph using concept of building of kinematic units, Mech. Mach. Theory, 134, 393-424, 2019b.

Shanmukhasundaram, V. R., Rao, Y. V. D., and Regalla, S. P.: Review of Structural Synthesis Algorithms for Epicyclic Gear Trains, Springer, Singapore, 2021.

Sun, W., Li, R., Kong, J., and Li, A.: Isomorphism Identification program code of Planetary Gear Trains, available at: https: //cloud.189.cn/t/Uryii2MVnmEz, last access: 8 February 2021 a.

Sun, W., Li, R., Kong, J., and Li, A.: Isomorphism Identification data of Planetary Gear Trains, available at: https://cloud.189.cn/ t/EJrEN36rQnUv, last access: 8 February 2021b.

Tsai, L.-W.: An Application of the Linkage Characteristic Polynomial to the Topological Synthesis of Epicyclic Gear Trains, J. Mech. Transm.-T ASME, 109, 329-336, 1987.

Tsai, L. W. and Lin, C. C.: The Creation of Nonfractionated, TwoDegree-of-Freedom Epicyclic Gear Trains, Journal is Journal of Mechanisms, Transmissions, and Automation in Design, 111, 524-529, https://doi.org/10.1115/1.3259033, 1989.

Xie, T., Hu, J., Peng, Z., and Liu, C.: Synthesis of seven-speed planetary gear trains for heavy-duty commercial vehicle, Mech. Mach. Theory, 90, 230-239, 2015a.

Xie, T., Hu, J., Peng, Z., and Liu, C.: Synthesis of seven-speed planetary gear trains for heavy-duty commercial vehicle, Mech. Mach. Theory, 90, 230-239, 2015b.

Xu, X., Sun, H., Liu, Y., and Dong, P.: Matrix-Based Operation Method for Detecting Structural Isomorphism of Plan- 
etary Gear Train Structures, J. Mech. Design, 142, 063301, https://doi.org/10.1115/1.4044916, 2020.

Yang, P., Pei, Z., Liao, N., and Yang, B.: Isomorphism identification for epicyclic gear mechanism based on mapping property and ant algorithm, Eng. Comput., 23, 49-54, 2007.

Yang, W. and Ding, H.: The Perimeter Loop-Based Method for the Automatic Isomorphism Detection in Planetary Gear Trains, J. Mech. Design, 140, 1-10, 2018a.

Yang, W. and Ding, H.: Automatic detection of degenerate planetary gear trains with different degree of freedoms, Appl. Math. Model., 64, 320-332, 2018b.
Yang, W. and Ding, H.: The Complete Set of One- Degree-ofFreedom Planetary Gear Trains With Up to Nine Links, J. Mech. Design, 141, 1-22, 2019.

Yang, W., Ding, H., Zi, B., and Zhang, D.: New graph representation for planetary gear trains, J. Mech. Design, 140, 1-10, 2018.

Yang, W., Ding, H., and Kecskeméthy, A.: Automatic synthesis of plane kinematic chains with prismatic pairs and up to 14 links, Mech. Mach. Theory, 132, 236-247, 2019. 